\title{
INFLUÊNCIA DAS DIMENSÕES DA ESCOVA E CREME DENTAL NA REALIZAÇÃO DA HIGIENE BUCAL EM CRIANÇAS DE 0 a 3 ANOS
}

\author{
INFLUENCE OF THE DIMENSIONS OF THE BRUSH AND DENTAL CREAM ON \\ THE PERFORMANCE OF THE ORAL HYGIENE IN CHILDREN 0-3 YEARS OLD
}

\author{
Flávio Salomão-Miranda*; Nataska Wanssa*; Karina Gerhardt Silva Bianco*; Victor \\ Hugo Bernardes de Oliveira*; Alcilene Aleixo da Cruz*
}

\section{Resumo}

A odontopediatria recomenda que as escovas dentais devem ter tamanho compatível com a idade do paciente e que o dentifrício deve ser fluoretado. A literatura atual indica que para as idades de 0-3 anos a quantidade de creme dental deve ser do tamanho de um grão de arroz. Este trabalho tem o objetivo de avaliar se as dimensões do creme e da escova dental podem influenciar na quantidade de creme dental utilizado para escovação em crianças de 0-3 anos. Quatro grupos foram criados: G1 - Escova adulta e Creme dental adulto; G2 - Escova adulta e Creme dental infantil; G3 - Escova infantil e Creme dental infantil e G4 - Escova Infantil e Creme dental Adulto. Os participantes recebiam escovas e cremes dentais de acordo com cada grupo e eram instruídos para colocarem a quantidade de creme dental para realizar a escovação de uma criança de 0-3 anos. Os resultados obtidos foram avaliados e submetidos aos testes de Análise de Variância e ao teste complementar de Tukey (5\%). Os resultados não demonstraram influencia estaticamente significativa das dimensões do creme e da escova na quantidade de dentifrício, entretanto, após a orientação educativa verificou-se diminuição na quantidade de creme dental colocados nas escovas. Independente do tamanho da escova ou creme dental fica clara a necessidade de orientação por parte dos cirurgiões dentistas quanto as quantidades corretas de creme dental que devem ser utilizadas para a escovação em crianças, principalmente as menores de 3 anos.

Palavras-chaves: Creme dental. Escova dental. Fluorose dentária.

\section{Abstract}

Pediatric dentistry recommends that toothbrushes should have a size compatible with the patient's age and that the toothpaste should be fluoridated. Current literature indicates that for ages 0-3 the amount of toothpaste should be the size of a grain of rice. This work aims to assess whether the dimensions of the toothpaste and

\footnotetext{
* CENTRO UNIVERSITÁRIO APARÍCIO CARVALHO - FIMCA
} 
toothbrush can influence the amount of toothpaste used for brushing in children aged 0-3 years. Four groups were created: G1 - Adult brush and Adult toothpaste; G2 Adult toothbrush and children's toothpaste; G3 - Children's toothbrush and toothpaste and G4 - Children's toothbrush and toothpaste. Participants received toothbrushes and toothpastes according to each group and were instructed to apply the amount of toothpaste to brush a 0-3 year old child. The results obtained were evaluated and submitted to the Analysis of Variance tests and to the complementary Tukey test $(5 \%)$. The results did not demonstrate a statistically significant influence of the dimensions of the cream and brush on the amount of toothpaste, however, after the educational guidance there was a decrease in the amount of toothpaste placed on the brushes. Regardless of the size of the brush or toothpaste, the need for guidance from dental surgeons regarding the correct amounts of toothpaste that should be used for brushing in children, especially those under 3 years old.

Keywords: Toothpaste. Toothbrush. Dental fluorosis.

\section{INTRODUÇÃO}

A remoção da placa dental apresenta papel central para a manutenção da saúde oral. A escolha adequada das escovas dentais e de quais dentifrícios utilizar auxiliam em um melhor resultado e em uma higienização mais adequada ${ }^{1}$.

A escova dentária, instrumento indispensável para a realização mecânica da higiene oral e a escolha de uma escova dental deve ser criteriosa e requer a observação de alguns fatores, como tamanho do cabo, ângulo entre a cabeça da escova e cabo, largura e comprimento da parte ativa, número de fileiras de cerdas, quantidade de cerdas por tufo. Além disso, suas dimensões estão relacionadas com a quantidade de dentifrício que as pessoas são influenciadas a utilizar, tanto nas crianças quanto nos adultos ${ }^{2}, 3$

A Associação Brasileira de Odontopediatria, recomenda que os dentifrícios fluoretados, tornem explícito nos rótulos a orientação de que seu uso seja feito com acompanhamento de um responsável, restringindo-o a quantidades pequenas. Crianças menores de quatro anos, por apresentarem maior probabilidade de ingestão dos dentifrícios pela falta de controle da deglutição, recomenda-se realizar a escovação com uma quantidade que equivale a um grão de arroz cru (aproximadamente $0,10 \mathrm{~g}$ ) com intuito de reduzir o risco para ocorrência de fluorose ${ }^{2}$. 
Apesar da recomendação do uso de dentifrícios fluoretados em crianças, os pacientes com pouca idade e com dificuldade motora exigem uma maior atenção por isso devem ser sempre auxiliados pelos responsáveis. O consumo máximo e indicado de flúor pelas crianças, onde previne-se a cárie dentária e diminui a ocorrência de fluorose dentária é de 0,05-0,07 mgF/ $/ \mathrm{Kg}$ peso/dia, sendo o creme dental o maior responsável pela ingestão de flúor ${ }^{4}$.

Existem diferentes fatores categóricas da ingestão de dentifrício fluoretado e do acontecimento da fluorose dentária, tais como, a idade em que se começa a realizar escovação dentaria, frequência de escovação e quantidade de dentifrício colocado na escova ${ }^{5}$.

Com a diversidade de escovas existentes, a escolha por um tipo que atenda às reais necessidades do usuário torna-se uma tarefa difícil quando o público não recebe informações adequadas. Portanto, a orientação de um profissional da Odontologia é fundamental ${ }^{3}$.

Não há dados na literatura que abordem a relação entre o tamanho do creme dental e da escova dental na quantidade de dentifrício utilizado.

\section{DESENVOLVIMENTO}

A perda dos dentes para maioria das pessoas ainda deriva, especialmente, da cárie e da doença periodontal. A cárie dental tem como fator etiológico o biofilme dental, cujo controle depende de uma boa higienização, a qual pode ser realizada por meios mecânicos, químicos ou a junção de ambos. É comprovado que como meio mecânico são mais efetivos para um adequando controle da placa, sendo a escova dental o recurso mais adequado e utilizado pelo paciente 6 .

As escovas dentárias apresentam quatro componentes básicos: cabo, haste ou intermediário, cabeça e cerdas. Segundo a Associação Dentária Americana $(\mathrm{ADA})^{7}$, a escova ideal deve apresentar certas características, tais como: tufos de cerdas com o mesmo comprimento, cabeça e haste situada no mesmo eixo, leveza e facilidade de limpeza, impermeabilidade à umidade, cerdas de nylon, cabeça pequena, baixo custo, durabilidade e eficiência no controle de placa.

Analisando as características morfológicas das escovas dentárias nacionais ${ }^{8}$ verificamos que as escovas dentárias deveriam apresentar características 
morfológicas tais como: cabo achatado, comprimento de cabeça de 25 a $32 \mathrm{~mm}$ e largura de 8 a $11 \mathrm{~mm}$, constassem tufos em fileiras de $3 \times 6$ ou $3 \times 8$, as cerdas fossem de nylon com comprimento uniforme, pontas arredondadas e de consistência média com diâmetro entre 0,30 a 0,32.

Não existem dados conclusivos que comprovem qual o desenho específico de escova dental ou forma que melhor remove a placa dental ${ }^{9}$, a sua escolha deve basear-se nas necessidades individuais de cada paciente e nas observações clínicas do profissional ${ }^{10}$.

Na primeira infância, é importante ressaltar que mais importante do que a técnica a ser executada é a frequência com que os dentes são escovados. É essencial durante esse procedimento uma boa visibilidade da cavidade bucal e o posicionamento seguro da criança, evitando assim movimentos bruscos e repentinos que poderiam feri-lo e predispor contra a escovação rotineira.

\section{METODOLOGIA}

A pesquisa foi aprovada pelo Comitê de Ética em Pesquisa sob número do parecer CAAE 97274718.3.0000.0012. Este trabalho foi realizado no Laboratório de Bioquímica do Centro Universitário Aparício Carvalho (UNIFIMCA).

O critério de inclusão dos participantes era ser maior de idade, ter filho(a) com idade menor que 3 anos e aceitar participar da pesquisa.

Inicialmente, os participantes foram abordados na recepção da clínica odontológica, onde após verificados os critérios de inclusão, foram convidados para participação da pesquisa e caso concordassem, deveriam assinar o termo de consentimento livre e esclarecido TCLE.

Os participantes $(n=8)$ foram encaminhados ao laboratório de Bioquímica, onde receberam escova e creme dental lacrados de acordo com os grupos descritos no quadro 1.

A pesquisa foi dividida em 02 etapas: a primeira, momento inicial onde 0 participante é instruído a colocar o creme dental na escova antes de receber orientação e a segunda, onde ele repete os passos realizados no primeiro momento, entretanto após a orientação educativa, conforme quadro 01. 
Quadro 1. Etapas da pesquisa

\begin{tabular}{|c|c|c|c|c|}
\hline \multicolumn{5}{|c|}{ Primeira Etapa: Antes da Orientação Educativa } \\
\hline \multirow{6}{*}{ 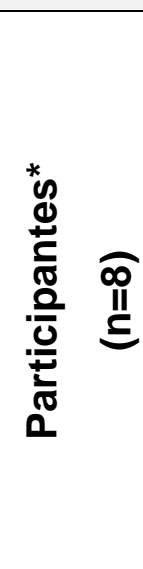 } & G1 & G2 & G3 & G4 \\
\hline & Escova & Escova & Escova & Escova \\
\hline & Adulta + & adulta + & Infantil + & Infantil+ \\
\hline & Creme & Creme & Creme & Creme \\
\hline & Adulto & Infantil & Infantil & Adulto \\
\hline & $\begin{array}{l}3 \text { amostras } \\
\text { (média e DP) }\end{array}$ & $\begin{array}{l}3 \text { amostras } \\
\text { (média e DP) }\end{array}$ & $\begin{array}{l}3 \text { amostras } \\
\text { (média e DP) }\end{array}$ & $\begin{array}{l}3 \text { amostras } \\
\text { (média e DP) }\end{array}$ \\
\hline \multicolumn{5}{|c|}{ Segunda Etapa: Após Orientação Educativa } \\
\hline \multirow{6}{*}{ 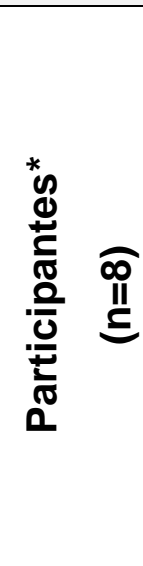 } & G1 & G2 & G3 & G4 \\
\hline & Escova & Escova & Escova & Escova \\
\hline & Adulta + & adulta + & Infantil + & Infantil+ \\
\hline & Creme & Creme & Creme & Creme \\
\hline & Adulto & Infantil & Infantil & Adulto \\
\hline & $\begin{array}{l}3 \text { amostras } \\
\text { (média e DP) }\end{array}$ & $\begin{array}{l}3 \text { amostras } \\
\text { (média e DP) }\end{array}$ & $\begin{array}{l}3 \text { amostras } \\
\text { (média e DP) }\end{array}$ & $\begin{array}{l}3 \text { amostras } \\
\text { (média e DP) }\end{array}$ \\
\hline
\end{tabular}

Os produtos utilizados na pesquisa foram: Creme dental infantil: Sorriso® Kids 50 gramas, Sabor Melancia Mágica (COLGATE-PAMOLIVE, SP, BRASIL) Lote: 9145BR122I e Creme dental adulto: Colgate ${ }^{\circledR} 180$ gramas Sabor Menta Refrescante (COLGATE-PAMOLIVE, SP, BRASIL) Lote: 9129BR123F. Escova dental infantil: Clean-B (China) REG./MS 25351.469157/2017-20 e Lote: DLCX032018; Escova adulta Clean-B (China) REG./MS 25351.469131/2017-26 e Lote: DLGB012018. As dimensões dos produtos utilizados estão dispostas no quadro 2 . 
Quadro 2. Características das escovas utilizados na pesquisa:

\begin{tabular}{|c|c|c|l|l|l|}
\hline Produto & Comprimento & Peso & $\begin{array}{l}\text { Número } \\
\text { total de } \\
\text { Tufos de } \\
\text { cerdas }\end{array}$ & $\begin{array}{l}\text { Material } \\
\text { das } \\
\text { Cerdas }\end{array}$ & $\begin{array}{l}\text { Área } \\
\text { superfici } \\
\text { al Total } \\
\text { das } \\
\text { Cerdas }\end{array}$ \\
\hline $\begin{array}{c}\text { Escova } \\
\text { dental } \\
\text { infantil: } \\
\text { Clean-B }\end{array}$ & $15 \mathrm{~cm}$ & 9,1 & 25 & nylon & $1,35 \mathrm{~cm}^{2}$ \\
\hline $\begin{array}{c}\text { Escova } \\
\text { adulta } \\
\text { Clean-B }\end{array}$ & $18,9 \mathrm{~cm}$ & 16,1 & 38 & nylon & $2,40 \mathrm{~cm}^{2}$ \\
gramas & & & & \\
\hline
\end{tabular}

Quadro 3. Características dos Dentifrícios utilizados na pesquisa:

\begin{tabular}{|c|c|c|c|c|}
\hline Produto & Comprimento & Peso & $\begin{array}{l}\text { Quantida } \\
\text { de de } \\
\text { Flúor }\end{array}$ & $\begin{array}{c}\text { Área } \\
\text { circular } \\
\text { da saída } \\
\text { do } \\
\text { dentifrício }\end{array}$ \\
\hline $\begin{array}{c}\text { Creme dental } \\
\text { infantil: Sorriso }{ }^{\circledR}\end{array}$ & $13,4 \mathrm{~cm}$ & $50 \mathrm{~g}$ & 1100 & $0,5024 \mathrm{~cm}$ \\
Kids & ppm & \\
\hline $\begin{array}{c}\text { Creme dental } \\
\text { adulto: Colgate }{ }^{\circledR}\end{array}$ & $19,5 \mathrm{~cm}$ & $180 \mathrm{~g}$ & 1450 & $0,5024 \mathrm{~cm}$ \\
\hline
\end{tabular}


Foto 1. Materiais utilizados para a realização das pesquisas
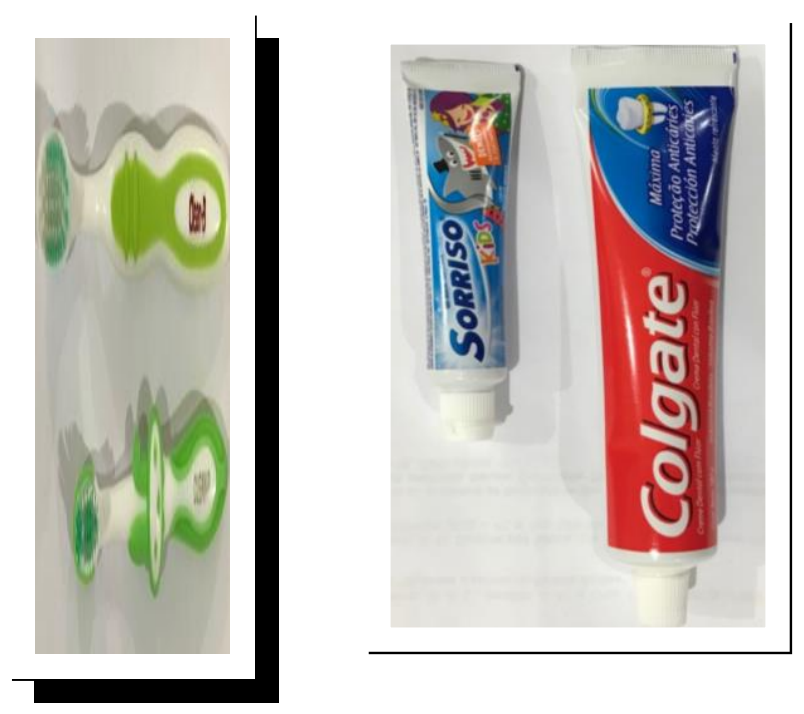

$\mathrm{Na}$ primeira etapa da pesquisa, os participantes abriram o creme dental e a escova recebida, eram instruídos a colocarem o creme dental na escova como se fossem realizar a higiene bucal de seus filhos. Cada combinação era repetida 03 vezes, a fim de se obter uma média para cada participantes em cada grupo.

Ao finalizar todas as combinações (G1, G2, G3 e G4) os participantes assistiam um vídeo onde era explicado a quantidade adequada para uma criança de 0 a 3 anos.

Para o cálculo da quantidade de creme dental utilizado, as escovas eram pesadas antes e após sua colocação do creme dental, utilizando-se balança de precisão BEL Engineering M5202 (Itália).

Todos os dados foram coletados e submetidos a análise estatística de ANOVA com repetição e Test T e através com nível de significância 5\%.

\section{RESULTADOS}

Após avaliação estatística, obtivemos os seguintes resultados de acordo com Tabela 1 e 2: 
Tabela 1. Média do peso e Desvio padrão de creme dental de acordo com os diferentes Grupos (antes da orientação educativa)

\begin{tabular}{l|l|l}
\hline Grupos & $\begin{array}{l}\text { Média do Peso } \\
\text { em Gramas }\end{array}$ & Desvio Padrão \\
\hline $\begin{array}{l}\text { G1: Escova Adulta + Creme } \\
\text { Adulto }\end{array}$ & $0,5083 \mathrm{~g}$ & 0,04389 \\
\hline $\begin{array}{l}\text { G2: Escova adulta + Creme } \\
\text { Infantil }\end{array}$ & $0,4125 \mathrm{~g}$ & 0,03307 \\
\hline $\begin{array}{l}\text { G3: Escova Infantil + Creme } \\
\text { Infantil }\end{array}$ & $0,3541 \mathrm{~g}$ & 0,00721 \\
\hline $\begin{array}{l}\text { G4: Escova Infantil+ Creme } \\
\text { Adulto }\end{array}$ & $0,3916 \mathrm{~g}$ & 0,00721 \\
\hline
\end{tabular}

$\mathrm{Na}$ tabela acima, percebemos que não há diferenças significativas entre os valores encontrados na primeira etapa, entretanto percebe-se maiores valores para os grupos com escova adulta (G1 e G2)

Os dados obtidos após a orientação educativa estão dispostos na tabela 2.

Tabela 2. Média do peso e Desvio padrão de creme dental de acordo com os diferentes Grupos (após palestra educativa)

\begin{tabular}{l|l|l}
\hline Grupos & $\begin{array}{l}\text { Média do Peso } \\
\text { em Gramas }\end{array}$ & Desvio Padrão \\
\hline $\begin{array}{l}\text { G1: Escova Adulta + Creme } \\
\text { Adulto }\end{array}$ & $0,1875 \mathrm{~g}$ & 0,01250 \\
\hline $\begin{array}{l}\text { G2: Escova adulta + Creme } \\
\text { Infantil }\end{array}$ & $0,2041 \mathrm{~g}$ & 0,03818 \\
\hline $\begin{array}{l}\text { G3: Escova Infantil + Creme } \\
\text { Infantil }\end{array}$ & $0,1875 \mathrm{~g}$ & 0,02165 \\
\hline $\begin{array}{l}\text { G4: Escova Infantil+ Creme } \\
\text { Adulto }\end{array}$ & $0,1875 \mathrm{~g}$ & 0,02165 \\
\hline
\end{tabular}

Também não foram encontradas diferenças significativas entre os resultados isoladamente encontrados após a orientação educativa, mas ainda podemos 
perceber que o grupo com escova dental adulta ainda manteve a maior média de peso em gramas (G2)

Quando relacionamos todas os dados obtidos antes e após a orientação educativa, podemos observar os resultados conforme tabela 3.

Tabela 3. Média do peso e Desvio padrão de creme dental de acordo com os diferentes Grupos Comparando Antes e após a palestra educativa

\begin{tabular}{l|l|l|l}
\hline Grupos & $\begin{array}{l}\text { Antes da } \\
\text { Palestra }\end{array}$ & $\begin{array}{l}\text { Após } \\
\text { Palestra }\end{array}$ & $\mathbf{P}<0,05$ \\
\hline $\begin{array}{l}\text { G1 Escova Adulta + Creme } \\
\text { Adulto }\end{array}$ & $0,5083 \mathrm{~g}$ & $0,1875 \mathrm{~g}$ & $*$ \\
\hline $\begin{array}{l}\text { G2 Escova adulta + Creme } \\
\text { Infantil }\end{array}$ & $0,4125 \mathrm{~g}$ & $0,2041 \mathrm{~g}$ & - -- \\
\hline $\begin{array}{l}\text { G3 Escova Infantil + Creme } \\
\text { Infantil }\end{array}$ & $0,3541 \mathrm{~g}$ & $0,1875 \mathrm{~g}$ & -- \\
\hline $\begin{array}{l}\text { G4 Escova Infantil+ Creme } \\
\text { Adulto }\end{array}$ & $0,3916 \mathrm{~g}$ & $0,1875 \mathrm{~g}$ & -- \\
\hline
\end{tabular}

${ }^{*}$ Diferença Estatisticamente Significante $(\mathrm{P}<0,05)$

Verificamos o efeito da orientação educativa na redução das médias do peso na quantidade de creme dental em todos os grupos, com diferença estatisticamente significativa para o primeiro grupo (G1).

\section{DISCUSSÃO}

Apesar de não se perceber estatisticamente a influência das dimensões do creme ou da escova nas quantidades de creme dental neste estudo, percebe-se que as maiores quantidades, tanto na primeira ou na segunda etapa, sempre estiveram presentes em grupos com Escova dental adulta (G1 e G2).

Possivelmente este resultado se deve as maiores dimensões da área de superfícies das cerdas, local onde se coloca o creme dental e devido ao fato comprimento total ser cerca de $31,29 \%$ maior do que a escova infantil. 
Tal hipótese já é comprovada em diversos setores, principalmente os da alimentação, onde pesquisas verificam a influência do tamanho da taça de vinho e copos de cerveja (recipientes) no aumento do consumo ${ }^{11}$ ou seja, aumentando-se o recipiente, existe uma tendência em aumento consumo do produto que se irá colocar no recipiente.

$\mathrm{Na}$ tabela 2 observamos uma distribuição quase uniforme dos pesos obtidos sem diferenças estatísticas entre si, entretanto com um discreto aumento na quantidade de creme dental quando utilizamos a escova de adulto (G2). O resultado encontrado pode ser explicado através da influência da educação em saúde, uma vez que os pais nesta ocasião receberam orientações sobre a quantidade adequada a ser utilizada, resultados semelhantes são encontrados estudos onde os pacientes que receberam orientação odontológica apresentaram maior percepção sobre a saúde bucal de seus filhos ${ }^{12}$.

Finalmente, os resultados apresentados a tabela 3 , demonstram que a palestra educacional foi capaz de produzir mudanças significativas (grupo 1) quando comparamos os momentos antes e após a palestra. Intervenções educativas realizadas por profissionais de saúde no contexto de sua prática apresentam potencial em promover a saúde bucal da população ${ }^{13}$.

\section{CONCLUSÃO}

No presente trabalho, não foi verificada a influência das dimensões das escovas ou dentifrícios na quantidade de creme dental utilizado para a realização da higiene bucal em crianças de 0 a 3 anos. Vale lembrar que existem centenas de marcas comerciais disponíveis no mercado com diferentes tamanhos de escovas e cremes dentais e por isso, mais pesquisas devem ser desenvolvidas neste sentido.

Independente do tamanho da escova ou creme dental fica clara a necessidade de orientação por parte dos cirurgiões dentistas quanto as quantidades corretas de creme dental que devem ser utilizadas para a escovação em crianças, principalmente as menores de 3 anos. 


\section{REFERÊNCIAS}

1 - Zaze ACSF ,Oliveira ER De, Melão MJAS, Alves E. Eficácia De Diferentes Tipos De Escovas Dentais Na Remoção Do Biofilme Bucal. Arq. Cienc. Saúde Unipar, Umuarama, V. 20, N. 2, P, 101-109, Maio/Ago. 2016.

2 - Roza PHJ, Studart LPC, Katz, CRT. Características dos dentifrícios infantis disponíveis no mercado brasileiro. Arq Odontol, Belo Horizonte. v.52 n. 4. p. 207-214, out/dez 2016.

3 - Bottan, E. R.; Campos, L.; Odebrecht, C. M. L. R.; Silveira, E. G.; Schmitt, P.; Araújo, M. S. Critérios adotados para a escolha da escova dental: estudo com consumidores de Florienópolis, Santa Catarina (BRASIL). Rev Sul-Brasileira de Odontologia (RSBO), 2009.

4- Burt, B. A. The changing patterns of systemic fluoride intake. Journal of Dental Research, [S.I.], v. 71, p. 1228-1237, 1992. (Special Issue).

5- Paiva, S. M.; Lima, Y. B.; Cury, J. A. Fluoride intake by Brazilian children from two communities with fluoridated water. Community Dentistry and Oral Epidemiology, [S.I.], v. 31, n. 3, p. 184-191, 2003.

6- Ripplinger, T.; Jagher, A. C.; Pinto, G. S.; Schardosim, L. R. Avaliação da utilização de dentifrício fluoretado em crianças. Rev RFO, Passo Fundo, v. 21, n. 1, p. 37-42, jan./abr., 2016.

7 -KUNERT, I.R. Estudo da ponta das cerdas das escovas em quarenta diferentes marcas. Rev Gaucha Odontol, v.40, n.4, p. 250- 254, jul./ago. 1992.

8- HALLA, D. A propósito das escovas dentárias. Rev. Paul. Odontol. v. 42, n. 2, p. 42-47, 1982.

9-STABBE, K. A. et al. A comparation de plaque reaccumulation and patient acceptance using a conventional toothbrush and a newly designed toothbrush. Clin. Prev. Dent. v. 10, n. 5, p. 10-14, 1988.

10 - BARROS, O.B. et al. Escovas dentais. Pós-Grad Rev Fac Odontol São José dos Campos, v.4, n.1, p. 33-8, jan./abr., 2001.

11- Pechey et al. Does wine glass size influence sales for on-site consumption? A multiple treatment reversal design. BMC Public Health 16:390, 2016.

12- Rigo L, Dalazen J, Garbin RR. Impacto da orientação odontológica para mães durante a gestação em relação à saúde bucal dos filhos. Einstein.14(2):219-25, 2016.

13- Menegaz, AM , Silva, ERA ,Cascaes AM. Intervenções educativas em serviços de saúde e saúde bucal: revisão sistemática. Rev Saude Publica.52:52; 2018. 